\title{
Designing periodic arrays of metal nanoparticles for light-trapping applications in solar cells
}

\author{
S. Mokkapati, ${ }^{1, a)}$ F. J. Beck, ${ }^{1}$ A. Polman, ${ }^{2}$ and K. R. Catchpole $^{1}$ \\ ${ }^{1}$ Center for Sustainable Energy Systems, College of Engineering and Computer Science, The Australian \\ National University, Canberra 0200, Australia \\ ${ }^{2}$ Center for Nanophotonics, FOM Institute AMOLF, Kruislaan 407, 1098 SJ Amsterdam, The Netherlands
}

(Received 30 April 2009; accepted 17 July 2009; published online 7 August 2009)

\begin{abstract}
We present criteria for optimizing the light-trapping efficiency of periodic arrays of metal nanoparticles for Si solar cell applications. The scattering cross section of the nanoparticles and the diffraction efficiency of the grating should be maximized in the long wavelength range. The grating pitch should be chosen to allow higher order diffraction modes for long wavelengths while maintaining the highest possible fill factor. These conditions place strong constraints on the optimal parameters (particle size of $\sim 200 \mathrm{~nm}$ and pitch of $\sim 400 \mathrm{~nm}$ ) for periodic arrays of metal nanoparticles, in contrast to dielectric gratings, where a relatively wide range of periods and feature sizes can be used for efficient light trapping. (C) 2009 American Institute of Physics.
\end{abstract}

[DOI: $10.1063 / 1.3200948]$

There is currently a lot of interest in silicon ( $\mathrm{Si}$ ) solar cells with thin active regions that minimize material costs while maintaining high efficiencies. Thin-film solar cells require efficient light trapping (LT) in order to increase the effective absorption length in the cell. LT is especially important at long wavelengths (close to the bandgap) in Si solar cells since $\mathrm{Si}$ is a poor absorber due to its indirect bandgap. Surface texturing, with feature sizes of a few microns, is routinely used for LT in wafer based Si solar cells ${ }^{1}$ but cannot be used in the case of thin active regions, which may themselves be of the order of a few microns thick. Light scattering by the excitation of localized surface plasmons in metal nanoparticles has been used to demonstrate effective LT for photovoltaic applications. ${ }^{2-4}$ Both random arrays ${ }^{3}$ and quasiperiodic arrays ${ }^{2}$ of metal nanoparticles have demonstrated significant absorption enhancement in solar cells. While there has been a systematic study on the requirements for efficient LT using random arrays, ${ }^{5,6}$ a range of additional parameters is introduced for periodic arrays of metal nanoparticles that need to be optimized. In this article, we study the effect of particle dimensions, the grating pitch, and fill factor and introduce essential criteria for optimizing the LT efficiency of periodic arrays of silver (Ag) nanoparticles. We demonstrate that for efficient LT, the optimal geometry of the metal nanoparticle array is relatively narrowly defined due to the limited range of particle sizes that result in efficient light scattering into the substrate in the long wavelength range. This is in contrast to dielectric gratings, where a relatively wide range of periods and feature sizes can be used for efficient $\mathrm{LT}^{7,8}$

Numerical simulations to estimate the LT efficiency of periodic arrays of nanoparticles were performed using LUMERICAL finite dimension time domain software. Semiinfinite $\mathrm{Si}$ substrates with periodic arrays of $\mathrm{Ag}$ nanoparticles on the surface are simulated in this study. Light is incident on the nanoparticle array from the Si side (Fig. 1). Only structures with $\mathrm{Ag}$ arrays on the rear have been simulated for this study, as Ag arrays on the front of the solar cell would result

${ }^{a)}$ Electronic mail: sudha.mokkapati@anu.edu.au. in lower external quantum efficiency compared to a cell without the nanoparticles at wavelengths below the plasmon resonance wavelength of the nanoparticles. ${ }^{9,10}$ A $20 \mathrm{~nm}$ thick $\mathrm{SiO}_{2}$ layer, typical of surface passivation layers used in $\mathrm{Si}$ solar cells, is also included between the Si substrate and the nanoparticles. An ordered array of Ag nanoparticles with a square base was simulated using square unit cell and periodic boundary conditions for the simulation volume. The particle height was $150 \mathrm{~nm}$. A plane wave source under normal incidence was used. The grating structure had equal periods in both $x$ - and $y$-directions, and light was incident in $z$ direction.

For an isolated particle, the scattering cross section provides a useful measure of interaction of light with the particle. The scattering cross section of the nanoparticle peaks at its plasmon resonance wavelength. However, for an array of particles, the scattering cross section is not defined. We therefore use absorption calculations to determine the plasmon resonance wavelength of the $\mathrm{Ag}$ nanoparticles in the periodic array since to first order, the absorption in the nanoparticles also peaks at the plasmon resonance wavelength. Absorption in Ag nanoparticles was determined from the difference in input and output power flows into a closed box surrounding the particle. Figure 2(a) shows the fraction of incident power absorbed in the $\mathrm{Ag}$ nanoparticle array for varying particle dimensions. The array pitch was fixed at 400 $\mathrm{nm}$. As can be seen, by increasing the particle size from 50 to $150 \mathrm{~nm}$, the plasmon resonance wavelength redshifts from $\sim 505$ to $\sim 770 \mathrm{~nm}$, while the absorption strength increases. The broadening of the absorption spectra with an increase in particle size is due to radiative damping and dynamic depo-

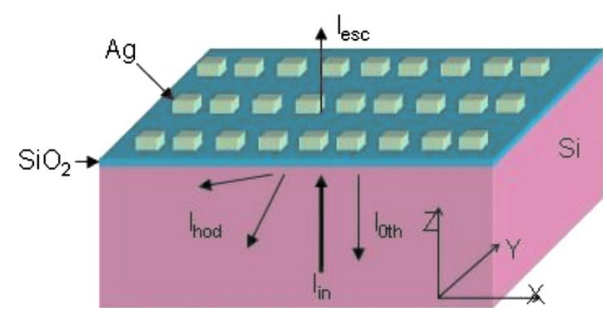

FIG. 1. (Color online) Schematic of the structures simulated for this study 


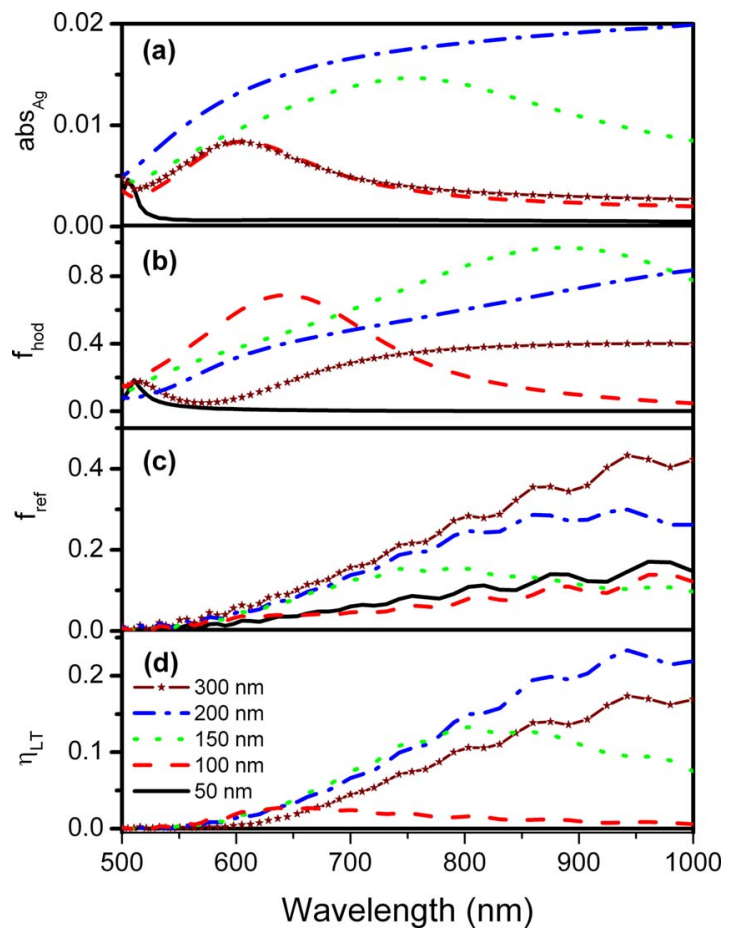

FIG. 2. (Color online) (a) Absorption in Ag nanoparticles, $\mathrm{abs}_{\mathrm{Ag}}$. (b) Fraction of light coupled into higher order diffraction modes in $\mathrm{Si}, f_{\text {hod }}$ (c) Fraction of incident source power reflected back into $\mathrm{Si}, f_{\text {ref }}$ (d) The LT efficiency, $\eta_{\mathrm{LT}}$, of a periodic array of $\mathrm{Ag}$ nanoparticles as a function of incident wavelength for different particle sizes. The particles have a square base with a height of $150 \mathrm{~nm}$. The lateral dimensions are indicated in the legend. The pitch of the grating is $400 \mathrm{~nm}$.

larization. The shift in the resonance wavelength is comparable to the resonance redshift for single particles (i.e., without periodic boundary conditions) with increasing size. The absorption in the nanoparticle array scales with the particle volume and the surface coverage. For particles with lateral dimensions of 200 and $300 \mathrm{~nm}$, the plasmon resonance wavelength is beyond $1000 \mathrm{~nm}$. For particle lateral dimensions of $300 \mathrm{~nm}$, an absorption peak at $\sim 580 \mathrm{~nm}$ is observed, which coincides with the absorption peak in the particle with base dimensions of $100 \mathrm{~nm}$. It is well known that as the metal nanoparticle size increases, its plasmon resonance wavelength corresponding to the dipole oscillations redshifts and higher order charge oscillations appear at shorter wavelengths. ${ }^{11}$ Based on this, we associate this peak with higher order charge oscillations in the $300 \mathrm{~nm}$ Ag nanoparticles.

After interaction with the nanoparticle array, a certain fraction of incident light is transmitted into air ( $I_{\text {esc }}$ in Fig. 1), and the rest is diffracted back into Si. The light diffracted back into $\mathrm{Si}$ is coupled into various allowed diffraction modes. The light coupled into the zeroth diffraction order, $I_{\text {oth }}$ in Fig. 1 (propagating along the direction of surface normal), is not trapped inside the substrate, as it lies within the escape cone of Si. Only light coupled into higher order diffraction modes, $I_{\text {hod }}$ in Fig. 1, which propagate outside the escape cone, is trapped inside Si. Figure 2(b) shows the light coupled into higher order diffraction modes in $\mathrm{Si}\left[f_{\text {hod }}\right.$ $\left.=I_{\text {hod }} /\left(I_{\text {hod }}+I_{\text {oth }}\right)\right]$ after interaction with the nanoparticle array as a fraction of total light coupled back into Si. As the particle size increases from 50 to $150 \mathrm{~nm}$, the wavelength range in which a large fraction of light is coupled into higher order diffraction modes redshifts. The largest $f_{\text {hod }}(\sim 96 \%)$ is obtained for a particle size of $150 \mathrm{~nm}$. For particle dimensions of $200 \mathrm{~nm}$, the wavelength range over which the maximum fraction of light is coupled into higher order diffraction modes lies beyond $1000 \mathrm{~nm}$. Clearly, a high fraction of light is coupled into higher order diffraction modes over a broad spectral range around the dipole resonance wavelengths [Fig. 2(a)]. So for efficient LT at wavelengths close to the bandgap of $\mathrm{Si}$, the particle dimensions should be chosen such that the plasmon resonance wavelength corresponding to the dipole charge oscillations in the particles lies in this wavelength range.

Figure 2(c) shows the fraction of incident power reflected into $\mathrm{Si}$, coupled into all diffraction orders, after interaction with the metal nanoparticle grating $\left[f_{\text {ref }}=\left(I_{\text {hod }}\right.\right.$ $\left.+I_{0 \mathrm{th}}\right) / I_{\text {in }}$ in Fig. 1]. In the long wavelength region, particles with dimensions of 200 and $300 \mathrm{~nm}$ have a higher $f_{\text {ref }}$ than particles with smaller dimensions. Figure 2(d) shows the net LT efficiency $\eta_{\mathrm{LT}}\left(=f_{\text {hod }} f_{\text {ref }}\right)$ for the arrays. It is clear that at wavelengths above $850 \mathrm{~nm}, \eta_{\mathrm{LT}}$ is lower for arrays of particles with base dimensions of $150 \mathrm{~nm}$ than for particles with base dimensions of 200 and $300 \mathrm{~nm}$, in spite of a higher fraction of scattered light going into higher order grating modes [Fig. 2(b)]. This is due to the smaller scattering cross section for the smaller particles. The data in Figs. 2(c) and 2(d) show oscillations that are ascribed to interference between light coupled into different diffraction modes.

The results in Fig. 2 indicate that for an ordered array of particles, a large fraction of light coupled into higher order diffraction modes $\left(f_{\text {hod }}\right)$ in the substrate by itself does not imply a high LT efficiency. The LT efficiency is determined by both the particle dimensions (which determine the plasmon resonance wavelength for an individual particle and hence $f_{\text {hod }}$ ) and the grating parameters (that determine $f_{\text {ref }}$ ). For efficient LT, it is essential to tune the grating parameters so that a high fraction of incident light is retained in $\mathrm{Si}$ at wavelengths around the particle plasmon resonance wavelength.

We now examine the effect of pitch and fill factor on the LT efficiency of an ordered array of Ag particles. For LT applications using periodic arrays of metal nanoparticles, at least one higher order diffraction mode should exist in $\mathrm{Si}$, with propagation angle of $>16^{\circ}$ with respect to the surface normal. Using the one-dimensional grating equation given by $\sin \theta_{p}=p \lambda / n L$, where $p$ is the order of the diffracted mode, $\lambda$ is the wavelength of incident light, $n$ is the refractive index of the substrate, $L$ is the pitch of the grating, and $\theta_{p}$ is the associated angle of propagation, the requirement of higher order propagating diffraction modes places a constraint on the minimum value of grating pitch that can be employed. In order for one higher order diffraction mode to exist at wavelength of $1000 \mathrm{~nm}$ in $\mathrm{Si}$, the grating pitch should be at least $300 \mathrm{~nm}$.

The net LT efficiency, $\eta_{\mathrm{LT}}$, as defined above, as a function of wavelength, for various grating pitches and a fixed particle size of $200 \mathrm{~nm}$ is depicted in Fig. 3. The curve for the $400 \mathrm{~nm}$ pitch is identical to that in Fig. 2(d). For a grating pitch of $250 \mathrm{~nm}$, long wavelength light is not trapped as no propagating higher order diffraction modes exist in Si. As the pitch is increased to $300 \mathrm{~nm}$, first order diffraction modes for longer wavelengths are allowed in Si and the light is trapped. The LT efficiency of the grating is further increased by in- 


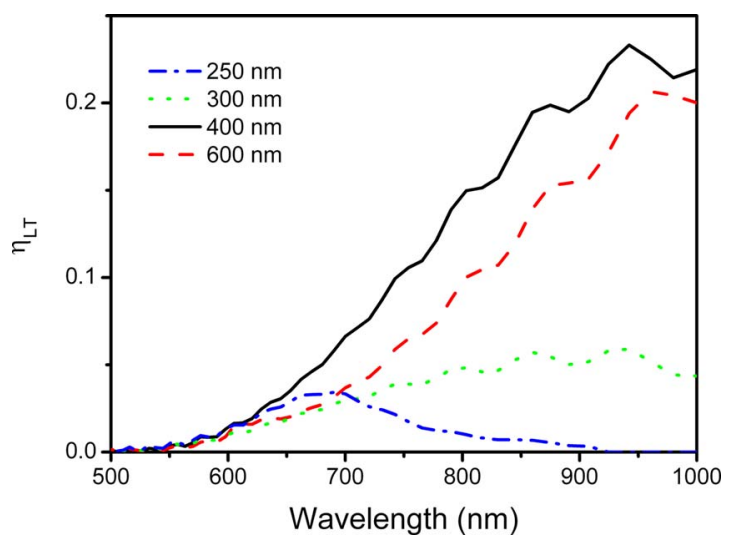

FIG. 3. (Color online) LT efficiency $\left(\eta_{\mathrm{LT}}\right)$ of a periodic array of Ag nanoparticles as a function of wavelength for different pitches (indicated in the legend). The particles have a square base with a side of $200 \mathrm{~nm}$ and a height of $150 \mathrm{~nm}$.

creasing the pitch to $400 \mathrm{~nm}$. This is because the propagation angles for the diffraction modes decrease as the pitch of the grating is increased. At a wavelength of $1000 \mathrm{~nm}$, the propagation angle for the first order diffraction modes in $\mathrm{Si}$ reduces from $\sim 64^{\circ}$ to $\sim 42.5^{\circ}$ by increasing the pitch from 300 to $400 \mathrm{~nm}$, resulting in an increase in $\eta_{\mathrm{LT}}$ by a factor of four. For diffraction orders with large propagation angles, there is poor impedance matching between the incident light, the allowed grating modes, and the diffraction modes, ${ }^{7}$ reducing the fraction of light coupled into these modes. As the propagation angle reduces (with increasing pitch), light is efficiently coupled into these diffraction modes. Once the propagation angles for higher order diffraction modes are relatively small, a further increase in the pitch of the grating results in reduced LT efficiency because of reduced fill factor (fraction of the unit cell area occupied by the nanoparticle). The scattering cross sections of isolated metal nanoparticles on a substrate, normalized to the particles geometrical cross section, are generally in the range from two to six; ${ }^{5}$ from this, we expect that a fill factor of at least $\sim 20 \%$ would be required for efficient light scattering. The effect of reducing fill factor is evident from Fig. 3 by comparing the LT efficiency for pitches of $400 \mathrm{~nm}$ (corresponding to a fill factor of $25 \%$ ) and $600 \mathrm{~nm}$ (fill factor of $11 \%$ ). These results indicate that for efficient LT applications, the pitch of the grating should be chosen such that one higher order diffraction mode exists in $\mathrm{Si}$ with reasonably small propagating angles while maintaining a sufficiently high fill factor.

In summary, for efficient LT using periodic arrays of metal nanoparticles, the particle dimensions and its dielectric environment should be chosen such that the dipole oscillation resonance lies in the wavelength range of interest. It is also necessary to tune the grating parameters so that high diffraction efficiency is obtained in a wavelength range close to the dipole oscillation resonance of the individual particles, ensuring that most of the incident power is retained in $\mathrm{Si}$. The pitch of the grating should be chosen to allow at least one diffraction mode propagating outside the escape cone in $\mathrm{Si}$ for long wavelength light. This imposes a lower limit of $\sim 400 \mathrm{~nm}$ on the grating pitch. In addition, a minimum fill factor of $\sim 20 \%$ should be maintained for efficient interaction between the incident light and the nanoparticle array. Taken together, these conditions place strong constraints on the optimal particle size and grating parameters for a periodic array, and based on these arguments, we conclude that arrays of particles with particle dimensions of $\sim 200 \mathrm{~nm}$ and a pitch of $\sim 400 \mathrm{~nm}$ are ideal for LT applications for Si solar cells.

The authors acknowledge the A. R. C. and NOW (for research conducted at the FOM as a part of the Joint Solar Programme) for financial support. We acknowledge SARA computing and networking services, supported by NWO.

${ }^{1}$ P. Campbell and M. A. Green, J. Appl. Phys. 62, 243 (1987).

${ }^{2}$ K. Nakayama, K. Tanabe, and H. A. Atwater, Appl. Phys. Lett. 93, 121904 (2008).

${ }^{3}$ K. R. Catchpole and S. Pillai, J. Appl. Phys. 100, 044504 (2006).

${ }^{4}$ S. Pillai, K. R. Catchpole, T. Trupke, and M. A. Green, J. Appl. Phys. 101, 093105 (2007).

${ }^{5}$ K. R. Catchpole and A. Polman, Appl. Phys. Lett. 93, 191113 (2008).

${ }^{6}$ K. R. Catchpole and A. Polman, Opt. Express 16, 21793 (2008).

${ }^{7}$ K. R. Catchpole, J. Appl. Phys. 102, 013102 (2007).

${ }^{8}$ K. R. Catchpole and M. A. Green, J. Appl. Phys. 101, 063105 (2007).

${ }^{9}$ S. H. Lim, W. Mar, P. Matheu, D. Derkacs, and E. T. Yu, J. Appl. Phys. 101, 104309 (2007).

${ }^{10}$ F. J. Beck, K. R. Catchpole, and A. Polman, J. Appl. Phys. 105, 114310 (2009).

${ }^{11}$ C. F. Bohren and D. R. Huffman, Absorption and Scattering of Light by Small Particles (Wiley-VHC, Weinheim, 2004). 